\title{
COHERENT RINGS OF FINITE WEAK GLOBAL DIMENSION
}

\author{
EDGAR E. ENOCHS, JUAN MARTÍNEZ HERNÁNDEZ, AND ALBERTO DEL VALLE
}

(Communicated by Wolmer V. Vasconcelos)

Dedicated to the memory of Professor Maurice Auslander

\begin{abstract}
The category of left modules over right coherent rings of finite weak global dimension has several nice features. For example, every left module over such a ring has a flat cover (Belshoff, Enochs, Xu) and, if the weak global dimension is at most two, every left module has a flat envelope (Asensio, Martínez). We will exploit these features of this category to study its objects.

In particular, we will consider orthogonal complements (relative to the extension functor) of several classes of modules in this category. In the case of a commutative ring we describe an idempotent radical on its category of modules which, when the weak global dimension does not exceed 2, can be used to analyze the structure of the flat envelopes and of the ring itself.
\end{abstract}

\section{INTRODUCTION}

Throughout this paper, $R$ is an associative ring with identity and module means left $R$-module. Flat covers and flat envelopes of modules have been extensively studied since they where introduced in [6], although the determination of the rings for which each module possess a flat cover (resp. envelope) is still an open problem. Nevertheless, we know that flat covers exist over right coherent rings of finite weak global dimension [3] and, restricting the value of that dimension to at most two, we have also guaranteed the existence of flat envelopes with the additional property that the associated diagrams can be completed in a unique way [2].

In Section 2, we use some properties of these covers and envelopes to study the modules $M$ such that $\operatorname{Ext}_{R}^{1}(M,-)\left(\right.$ resp. $\left.\operatorname{Ext}_{R}^{1}(-, M)\right)$ vanishes over the class $\mathfrak{F}$ of all flat modules. The modules $M$ with $\operatorname{Ext}_{R}^{1}(\mathfrak{F}, M)=0$, called cotorsion, have been successfully used in the study of flat covers ([3],[14]), and our main concern in this paper will be with the condition $\operatorname{Ext}_{R}^{1}(M, \mathfrak{F})=0$ and its relation to flat envelopes. In this way, we consider in Section 3 the modules $M$ such that $\operatorname{Hom}_{R}(M, \mathfrak{F})=0$ and $\operatorname{Ext}_{R}^{1}(M, \mathfrak{F})=0$ (called $\varphi$-torsion modules). For commutative coherent rings of finite weak global dimension, they form a torsion class which is shown to be hereditary when the weak global dimension does not exceed 2. This is done in Section 4, where we use this fact to show that monomorphic flat envelopes must be essential. If the ring is furthermore local, a description is given of the flat envelopes

Received by the editors February 1, 1996 and, in revised form, November 19, 1996.

1991 Mathematics Subject Classification. Primary 13C11, 13D05, 16D40, 16E70.

Key words and phrases. Coherent ring, weak global dimension, flat envelope.

The second and third authors are supported by the DGICYT of Spain (PB93-0515-C02-02) and by the Comunidad Autónoma de la Región de Murcia (PIB94/25). 
of ideals in terms of greatest common divisors of their elements, which, together with the previous results, is employed to give some light in the description of the structure of such rings.

Troughout the paper we write $\mathrm{wd}(R)$ for the weak global dimension of the ring $R$ and $\operatorname{fd}_{R}(L)$ for the flat dimension of the module $L$. Also, we shall denote the category of left $R$-modules by ${ }_{R} \operatorname{Mod}$ and write $\operatorname{Ext}_{R}(A, B)$ instead of $\operatorname{Ext}_{R}^{1}(A, B)$.

\section{Orthogonal COMPLEMENTS}

Let $M$ be a module. A flat preenvelope of $M$ is a homomorphism $f: M \rightarrow F$ such that the module $F$ is flat and, for any other homomorphism $g: M \rightarrow G$ with $G$ flat, there exists a third homomorphism $h: F \rightarrow G$ such that $g=h \circ f$. If, moreover, any endomorphism $h$ of $F$ such that $f=h \circ f$ is an automorphism of $F$, then $f$ is said to be a flat envelope of $M$. A flat envelope need not be a monomorphism; when it is one, we speak about a monomorphic flat envelope. Dually, one can define a flat (pre) cover of $M$, which must be an epimorphism. These concepts were defined in $[6]$.

If $\mathcal{C}$ is a class of modules such that $\operatorname{Hom}_{R}(M, C)=0\left(\operatorname{resp} \operatorname{Hom}_{R}(C, M)=0\right)$ for all $C \in \mathcal{C}$, then we shall write $\operatorname{Hom}_{R}(M, \mathcal{C})=0\left(\operatorname{resp} . \operatorname{Hom}_{R}(\mathcal{C}, M)=0\right)$. Similar conventions will be used for the bifunctor $\operatorname{Ext}_{R}(-,-)$. The class of all modules $M$ with $\operatorname{Ext}_{R}(M, \mathcal{C})=0$ (resp. $\left.\operatorname{Ext}_{R}(\mathcal{C}, M)=0\right)$ will be denoted by ${ }^{\perp} \mathcal{C}$ (resp. $\mathcal{C}^{\perp}$ ). This is usually called the left (resp. right) orthogonal complement (relative to the functor $\operatorname{Ext}_{R}(-,-)$ ) of the class $\mathcal{C}$.

When $\mathfrak{F}$ is the class of all flat left $R$-modules, the objects of $\mathfrak{F}^{\perp}$ are the cotorsion modules as defined in [7]; these were later used by Belshoff, Enochs and Xu in the determination of two wide classes of rings over which each module has a flat cover ([3],[14]). The relevant class of modules in this paper, however, is a subclass of ${ }^{\perp} \mathfrak{F}$ which is intimately related with flat envelopes. Its study will be carried out in the next section, while the present one is devoted to preliminary results and to a brief discussion of the double orthogonal classes ${ }^{\perp}\left(\mathfrak{F}^{\perp}\right)$ and $\left({ }^{\perp} \mathfrak{F}\right)^{\perp}$.

We start with a result (Corollary 2.2) dual to [7, Lemma 2.2] that is deduced here from the more general Proposition 2.1, which may have independent interest.

Proposition 2.1. Let $f: M \rightarrow F$ be a flat envelope, $G$ a flat module, and suppose that the following diagram is commutative with exact row:

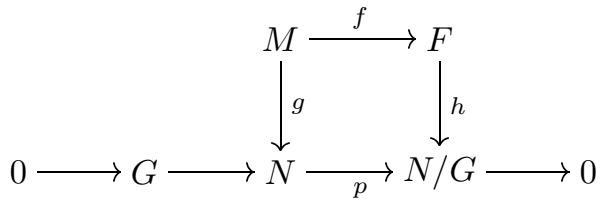

Then there exists a homomorphism $q: F \rightarrow N$ such that $p \circ q=h$ and $q \circ f=g$.

Proof. The pull-back $\left\{P ; p^{\prime}, h^{\prime}\right\}$ of $p, h$ gives a commutative diagram with exact rows

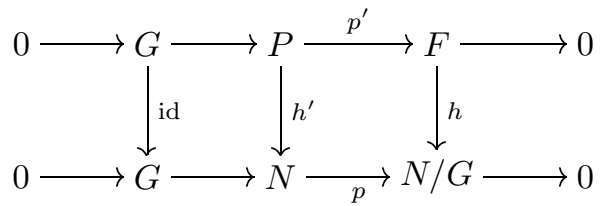


which shows that $P$ is a flat module. By the universal property of pull-backs there is a map $s: M \rightarrow P$ such that $h^{\prime} \circ s=g$ and $p^{\prime} \circ s=f$. Since $f$ is a flat envelope of $M$, we have $t: F \rightarrow P$ such that $t \circ f=s$. Hence $p^{\prime} \circ t \circ f=f$, and thus $p^{\prime} \circ t$ is an automorphism. The desired homomorphism is then $q=h^{\prime} \circ t \circ\left(p^{\prime} \circ t\right)^{-1}$.

Corollary 2.2. If $f: M \rightarrow F$ is a flat envelope then $\operatorname{Coker}(f) \in{ }^{\perp} \mathfrak{F}$.

Proof. Let $\pi: F \rightarrow C$ be the cokernel of $f$ and let $p: N \rightarrow C$ be an epimorphism with flat kernel. Then, by Proposition 2.1, there exists a homomorphism $q: F \rightarrow N$ such that $p \circ q=\pi$ and $q \circ f=0$. Thus $p$ is a retraction and hence $\operatorname{Ext}_{R}(C, \mathfrak{F})=$ 0 .

Next we consider the double orthogonal classes ${ }^{\perp}\left(\mathfrak{F}^{\perp}\right)$ and $\left({ }^{\perp} \mathfrak{F}\right)^{\perp}$, which clearly lie between $\mathfrak{F}$ and ${ }_{R}$ Mod. We discuss here the two extreme possibilities for them, i.e. when they coincide with $\mathfrak{F}$ or with ${ }_{R}$ Mod. In this direction we have the following.

Proposition 2.3. Let $R$ be a ring such that each left $R$-module has a flat cover. Then ${ }^{\perp}\left(\mathfrak{F}^{\perp}\right)=\mathfrak{F}$.

Proof. Let $M \in{ }^{\perp}\left(\mathfrak{F}^{\perp}\right)$ and let $f: F \rightarrow M$ be a flat cover of $M$; from [7, Lemma 2.2] we see that $K=\operatorname{Ker}(f) \in \mathfrak{F}^{\perp}$, and so $f$ is split and $M$ is flat.

Corollary 2.4. If $R$ is a ring such that each left $R$-module has a flat cover, then ${ }^{\perp}\left(\mathfrak{F}^{\perp}\right)={ }_{R} \operatorname{Mod}$ if and only if $R$ is von Neumann regular.

It is easy to see that $\left({ }^{\perp} \mathfrak{F}\right)^{\perp}={ }_{R}$ Mod if and only if ${ }^{\perp} \mathfrak{F}=\mathfrak{P}$ (the class of projective modules). In particular, this occurs when the weak global dimension of $R$ is at most 1. For the case $\left({ }^{\perp} \mathfrak{F}\right)^{\perp}=\mathfrak{F}$, we recall that a left $I F$-ring is a ring whose injective left modules are flat, and that a module $G$ is said to be FP-injective if, for every exact sequence $0 \rightarrow L \rightarrow M \rightarrow N \rightarrow 0$ with $N$ finitely presented, each homomorphism $L \rightarrow G$ can be extended to $M[12$, p. 28].

Theorem 2.5. The following conditions hold:

(1) If every left $R$-module has a monomorphic flat envelope or if $R$ is a two-sided IF-ring, then $\left({ }^{\perp} \mathfrak{F}\right)^{\perp}=\mathfrak{F}$.

(2) If $\left({ }^{\perp} \mathfrak{F}\right)^{\perp}=\mathfrak{F}$, then $R$ is a right coherent left IF ring (equivalently, a ring whose left modules have monomorphic flat preenvelopes).

Proof. (1) The first part is dual to Proposition 2.3, by Corollary 2.2. For the second, if $R$ is IF in both sides, then the class of all flat modules coincides with the class of all FP-injective modules [8, Proposition 2.3], and hence the class $\mathfrak{F P}$ of finitely presented modules is contained in ${ }^{\perp} \mathfrak{F}$, whence $\left({ }^{\perp} \mathfrak{F}\right)^{\perp} \subseteq(\mathfrak{F P})^{\perp}=\mathfrak{F}$.

(2) It is clear that $R$ is left IF, while the right coherence of $R$ follows from the isomorphisms $\operatorname{Ext}_{R}\left(C, \Pi F_{\alpha}\right) \cong \operatorname{\Pi Ext}_{R}\left(C, F_{\alpha}\right)$. The statement in parentheses follows from [6, Proposition 5.1].

In case our ring is commutative, we get that $\left({ }^{\perp} \mathfrak{F}\right)^{\perp}=\mathfrak{F}$ if and only if $R$ is an IF-ring. But, in general, neither of the implications in (1) admits a converse, since we know of examples of rings with monomorphic flat envelopes which are not two-sided IF [9, Example 2] and of (commutative) IF-rings without flat envelopes $[1$, p. 435]. It remains as an open question whether the converse of (2) is valid. 


\section{3. $\varphi$-TORSION MODULES}

We know that cokernels of flat envelopes are in ${ }^{\perp} \mathfrak{F}$, although the converse is clearly not true (as any nonzero projective module shows). Our main interest in what follows will be on right coherent rings of weak global dimension at most 2, that is, rings for which every left module has a flat envelope where the associated diagrams can be completed in a unique way [2, Proposition 2.1]. The following result is the motivation for our definition of $\varphi$-torsion modules.

Lemma 3.1. For a module $T$ the following conditions are equivalent:

(a) $\operatorname{Hom}_{R}(T, \mathfrak{F})=0$ and $\operatorname{Ext}_{R}(T, \mathfrak{F})=0$;

(b) $T$ is the cokernel of a (monomorphic) flat envelope which completes the diagrams in a unique way.

Proof. Assume (a) and let $0 \rightarrow M \rightarrow F \rightarrow T \rightarrow 0$ be any exact sequence with $F$ flat. We get an induced isomorphism $\operatorname{Hom}_{R}\left(F, F^{\prime}\right) \rightarrow \operatorname{Hom}_{R}\left(M, F^{\prime}\right)$ for each flat module $F^{\prime}$, from which (b) follows. Conversely, if (b) holds and $f: M \rightarrow F$ is the given envelope, then $\operatorname{Hom}_{R}\left(f, F^{\prime}\right)$ is monic for each $F^{\prime}$ flat and so $\operatorname{Hom}_{R}(T, \mathfrak{F})=0$, while $\operatorname{Ext}_{R}(T, \mathfrak{F})=0$ follows from Corollary 2.2.

We call a module $M \varphi$-torsion if it satisfies the equivalent conditions of Lemma 3.1. Over a right coherent ring of weak global dimension at most 2 the $\varphi$-torsion modules are exactly the cokernels of flat envelopes.

Remark 3.1. The classical torsion modules over a domain $R$ are those modules $T$ that satisfy $\operatorname{Hom}_{R}(T, \mathfrak{F})=0$, and thus every $\varphi$-torsion module is torsion, but the converse is not true in general. For example, if $\operatorname{wd}(R) \leq 1$, then 0 is the only $\varphi$-torsion module. If $R=k[[x, y]]$ with $k$ a field, then since the inclusion of the maximal ideal $(x, y)$ into $R$ is a flat envelope, $R /(x, y)$ is $\varphi$-torsion. Hence all artinian $R$-modules are $\varphi$-torsion. But $R /(x)$ is torsion but not $\varphi$-torsion, since $(x)$ is flat and the exact sequence $0 \rightarrow(x) \rightarrow R \rightarrow R /(x) \rightarrow 0$ does not split.

For an arbitrary ring $R$, it is clear that the class of $\varphi$-torsion modules is closed under extensions, direct summands and direct sums. We recall that a class $\mathcal{T}$ of modules is a torsion class if it is closed under extensions, direct sums and factor modules. In this sense, note that if $0 \rightarrow C^{\prime} \rightarrow C \rightarrow C^{\prime \prime} \rightarrow 0$ is an exact sequence with $C \varphi$-torsion and $F$ is a flat module, then we see from the exact sequence

$$
\begin{aligned}
0 \rightarrow \operatorname{Hom}_{R}\left(C^{\prime \prime}, F\right) & \rightarrow \operatorname{Hom}_{R}(C, F) \rightarrow \operatorname{Hom}_{R}\left(C^{\prime}, F\right) \\
& \rightarrow \operatorname{Ext}_{R}\left(C^{\prime \prime}, F\right) \rightarrow \operatorname{Ext}_{R}(C, F)
\end{aligned}
$$

that

$$
\operatorname{Hom}_{R}\left(C^{\prime \prime}, F\right)=0 \text { and } \operatorname{Hom}_{R}\left(C^{\prime}, F\right) \cong \operatorname{Ext}_{R}\left(C^{\prime \prime}, F\right),
$$

so that the $\varphi$-torsion modules form a torsion class if and only if $\operatorname{Hom}_{R}\left(C^{\prime}, \mathfrak{F}\right)=0$ for any submodule $C^{\prime}$ of a $\varphi$-torsion module. Next we look for rings satisfying this condition.

Proposition 3.2. Let $R$ be a commutative ring such that, for each maximal ideal $P$ of $R$, the injective envelope (as $R$-module) of each flat $R_{P}$-module is flat, and let $C$ be an $R$-module such that $\operatorname{Hom}_{R}(C, \mathfrak{F})=0$. Then $\operatorname{Hom}_{R}\left(C^{\prime}, \mathfrak{F}\right)=0$ holds for every submodule $C^{\prime}$ of $C$. 
Proof. Let $C^{\prime}$ be a submodule of $C$ and let $F$ be a flat $R$-module; we must show that $\operatorname{Hom}_{R}\left(C^{\prime}, F\right)=0$. Now, for a maximal ideal $P$ of $R$, the injective envelope $\widehat{F_{P}}$ of $F_{P}$ is flat and hence $\operatorname{Hom}_{R}\left(C, \widehat{F_{P}}\right)=0$, whereas $\operatorname{Hom}_{R}\left(C^{\prime}, \widehat{F_{P}}\right)=0$ and hence

$$
\operatorname{Hom}_{R}\left(C^{\prime}, F\right) \subseteq \operatorname{Hom}_{R}\left(C^{\prime}, \prod F_{P}\right) \subseteq \operatorname{Hom}_{R}\left(C^{\prime}, \prod \widehat{F_{P}}\right) \cong \prod \operatorname{Hom}_{R}\left(C^{\prime}, \widehat{F_{P}}\right)=0,
$$

where the product runs over all the maximal ideals $P$ of $R$.

Corollary 3.3. Over a commutative ring $R$ satisfying the conditions of Proposition 3.2, the $\varphi$-torsion modules form a torsion class.

Remark 3.2. Gorenstein rings and rings whose localizations at maximal ideals are domains satisfy the conditions of Proposition 3.2. In particular, all commutative coherent rings of finite weak global dimension do [13, Theorem 5.1].

\section{Coherent rings of Weak gLobal Dimension two}

Troughout this section, $R$ will be a commutative and coherent ring of weak global dimension at most 2 . We plan to show that the class of $\varphi$-torsion modules is hereditary, and derive from this that all monomorphic flat envelopes over $R$ are essential monomorphisms (in this case we speak about essential flat envelopes). Also, an instance of local coherent rings of weak global dimension 2 whose prime spectrum looks like the spectrum of an umbrella ring will be presented.

We shall use results about torsion theories and the associated (noncommutative) localization in ${ }_{R}$ Mod. The required background, as well as the notation employed, will be introduced as needed. For details, we direct the reader to [12].

Let $\phi$ be an idempotent radical in ${ }_{R}$ Mod, that is, a subfunctor of the identity functor in ${ }_{R}$ Mod such that $\phi \circ \phi=\phi$ and $\phi(M / \phi(M))=0$ for each module $M$. The modules $T$ for which $\phi(T)=T$ are called $\phi$-torsion, and the class $\mathcal{T}(\phi)$ of all $\phi$ torsion modules is a torsion class. The modules $L$ with $\phi(L)=0$ are $\phi$-torsionfree, and the class $\mathcal{F}(\phi)$ of all $\phi$-torsionfree modules is a torsionfree class, i.e. it is closed under extensions, direct products and submodules. $\mathcal{T}(\phi)$ determines $\mathcal{F}(\phi)$ in the sense that $L \in \mathcal{F}(\phi)$ if and only if $\operatorname{Hom}_{R}(\mathcal{T}(\phi), L)=0$, and also determines $\phi$ since $\phi(M)=\sum\{T \leq M \mid T \in \mathcal{T}(\phi)\}$ for each module $M$.

Consistently with the above, we shall write $\varphi$ for the idempotent radical associated to the class $\mathcal{T}(\varphi)$ of all $\varphi$-torsion modules. We get:

Proposition 4.1. $\mathcal{F}(\varphi)=\left\{L \in{ }_{R} \operatorname{Mod}: \operatorname{fd}(L) \leq 1\right\}$.

Proof. We show that $\mathrm{fd}(L) \leq 1$ if and only if $\operatorname{Hom}_{R}(\mathcal{T}(\varphi), L)=0$. Fix an exact sequence $0 \rightarrow K \rightarrow F \rightarrow L \rightarrow 0$ with $F$ flat. If $\mathrm{fd}(L) \leq 1$ and $h: T \rightarrow L$ is a homomorphism with $T \in \mathcal{T}(\varphi)$, then the upper row of the following diagram (in which the right-hand square is a pullback) splits, so that $h$ factors through a homomorphism $T \rightarrow F$ and therefore must be zero:

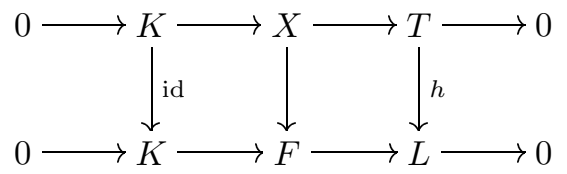


On the other hand, if $\operatorname{Hom}_{R}(\mathcal{T}(\varphi), L)=0$, take a flat envelope $K \rightarrow G$ of $K$ with cokernel $T$ and complete the diagram below with $g$ :

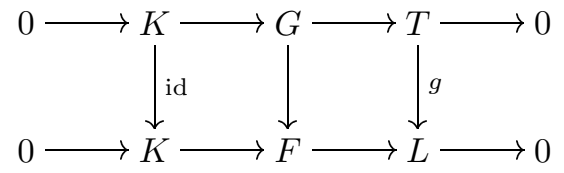

As $T \in \mathcal{T}(\varphi)$, we must have $g=0$, so the upper row splits and $K$ is flat.

The following result clearly shows that it is enough to study the left exactness of $\varphi$ for local rings. Note that the assumptions on $R$ are preserved by localization.

Proposition 4.2. For any $R$-module $T$ the following conditions are equivalent:

(a) $T$ is a $\varphi$-torsion $R$-module;

(b) $T_{P}$ is a $\varphi$-torsion $R_{P}$-module for each prime ideal $P$ of $R$;

(c) $T_{M}$ is a $\varphi$-torsion $R_{M}$-module for each maximal ideal $M$ of $R$.

Proof. (a) $\Rightarrow$ (b). Let $T$ be a $\varphi$-torsion $R$-module, $P$ a prime ideal, and take an exact sequence $0 \rightarrow M \rightarrow F \rightarrow T \rightarrow 0$ in which $M \rightarrow F$ is a flat envelope. It is then routine to check that the induced map $M_{P} \rightarrow F_{P}$ is a flat envelope of $M_{P}$ as an $R_{P}$-module, and thus its cokernel $T_{P}$ is $\varphi$-torsion.

(b) $\Rightarrow$ (c). This is trivial.

(c) $\Rightarrow$ (a). Let $T$ be as in (c) and let $L$ be an $R$-module with $\operatorname{fd}_{R}(L) \leq 1$; then $\mathrm{fd}_{R_{M}}\left(L_{M}\right) \leq 1$ for each maximal ideal $M$, and so $\operatorname{Hom}_{R_{M}}\left(T_{M}, L_{M}\right)=0$, which clearly implies $\operatorname{Hom}_{R}(T, L)=0$, so that $T$ is $\varphi$-torsion by Proposition 4.1.

Next, we study how to construct the monomorphic flat envelopes of modules. Later on, it will be shown that these are in fact essential monomorphisms.

Proposition 4.3. Let $M$ be a submodule of a flat module $F^{\prime}$. Then there exists a unique minimal flat submodule $F$ of $F^{\prime}$ containing $M$, and the inclusion map $M \hookrightarrow F$ is a flat envelope.

Proof. As inverse limits of flat $R$-modules are flat [12, p. 100], $F$ is just the intersection of all flat submodules of $F^{\prime}$ containing $M$. Now take a flat envelope $M \rightarrow G$ of $M$ and complete the diagram with exact rows below with $\alpha$ and $\beta$ :

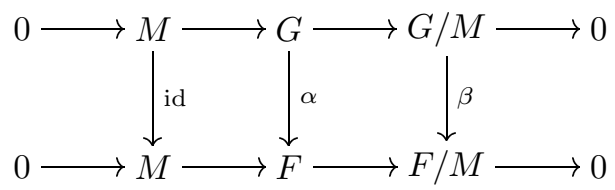

Then $\operatorname{Ker}(\alpha) \cong \operatorname{Ker}(\beta)$ is flat and hence its inclusion into $G / M$ splits, so that $\operatorname{Ker}(\alpha)=0$; and thus, by the minimality of $F, \alpha$ is an isomorphism.

If $R$ is a local ring then, by [13, Theorem 5.21], it is a GCD-domain, that is a domain in which each finite set of elements $a_{1}, \ldots, a_{n}$ possess a greatest common divisor $\left[a_{1}, \ldots, a_{n}\right]$. We can describe the flat envelopes of ideals of $R$ as follows:

Proposition 4.4. Let $R$ be local. Then:

(1) An ideal $F$ of $R$ is flat if and only if $\left[a_{1}, \ldots, a_{n}\right] \in F$ for all $a_{1}, \ldots, a_{n} \in F$.

(2) The flat envelope of an ideal $I$ of $R$ is its inclusion into the direct union of the principal ideals of the form $R\left[a_{1}, \ldots, a_{n}\right]$, where $a_{1}, \ldots, a_{n} \in I$. 
(3) The flat envelope of the ideal $R a_{1}+\cdots+R a_{n}$ is its inclusion into $R\left[a_{1}, \ldots, a_{n}\right]$.

Proof. By [11, Corollary 1.3] the flat ideals of $R$ are exactly the directed unions of principal ideals, and (1) follows easily from this observation. For (2), note that the set of principal ideals $\left\{R\left[a_{1}, \ldots, a_{n}\right]: a_{1}, \ldots, a_{n} \in I\right\}$ is directed by inclusion, and so its direct union $F(I)$ is flat; it is the flat envelope of $I$ by Proposition 4.3 since, for any flat ideal $G$ containing $I$, we get $\left[a_{1}, \ldots, a_{n}\right] \in G$ for all $a_{1}, \ldots, a_{n} \in I$ by (1) and hence $F(I) \subseteq G$. (3) is now obvious.

Given an idempotent radical $\phi$ in ${ }_{R}$ Mod, we say that a (left) ideal $I$ of $R$ is $\phi$-dense if the cyclic module $R / I$ is $\phi$-torsion; the set $\mathcal{G}(\phi)$ of all $\phi$-dense ideals is a filter in the lattice of left ideals of $R$. We have:

Corollary 4.5. If $R$ is local then $\mathcal{G}(\varphi)$ has a basis of finitely generated ideals.

Proof. From Proposition 4.3 and the proof of Lemma 3.1 it follows that $I \in \mathcal{G}(\varphi)$ if and only if $I \hookrightarrow R$ is a flat envelope. In this case, by Proposition 4.4, we must have $\left[a_{1}, \ldots, a_{n}\right]=1$ for some $a_{1}, \ldots, a_{n} \in I$, and thus $R a_{1}+\cdots+R a_{n} \in \mathcal{G}(\varphi)$.

An idempotent radical $\phi$ is a left exact functor (then called a left exact radical) if and only if $\mathcal{T}(\phi)$ is hereditary, i.e. closed under submodules. $\mathcal{G}(\phi)$ is then (the filter associated to) a Gabriel topology in $R$ (see [12, p. 146]). Gabriel topologies and left exact radicals in ${ }_{R}$ Mod determine each other. When $R$ is commutative, $\mathcal{G}(\phi)$ is a Gabriel topology in $R$ even if $\phi$ is not left exact [4, p. 88].

We introduce now two left exact radicals in ${ }_{R}$ Mod related to $\varphi$ (which, a posteriori, will coincide with it). One of them is the unique left exact radical $\tau$ such that $\mathcal{G}(\tau)=\mathcal{G}(\varphi)$; this is the biggest left exact radical smaller than $\varphi$. The other is the radical $\sigma$ whose associated torsion class is generated by the submodules of $\varphi$-torsion modules; this is the smallest left exact radical bigger than $\varphi$.

Associated with each idempotent radical $\phi$ in ${ }_{R}$ Mod we define

$$
\begin{aligned}
& \mathcal{Z}(\phi)=\{P \in \operatorname{Spec}(R): R / P \text { is } \phi \text {-torsion }\}=\mathcal{G}(\phi) \cap \operatorname{Spec}(R), \\
& \mathcal{K}(\phi)=\{P \in \operatorname{Spec}(R): R / P \text { is } \phi \text {-torsionfree }\} .
\end{aligned}
$$

If $\phi$ is left exact they constitute a partition of the prime spectrum $\operatorname{Spec}(R)$ and $\mathcal{K}(\phi)$ is generically closed, that is, $P \in \mathcal{K}(\phi), Q \in \operatorname{Spec}(R)$ and $Q \subseteq P$ imply $Q \in \mathcal{K}(\phi)$. Conversely, for any generically closed subset $\Omega$ of $\operatorname{Spec}(R)$ we find a left exact radical $\xi_{\Omega}$ whose torsion class is

$$
\mathcal{T}\left(\xi_{\Omega}\right)=\left\{M \in{ }_{R} \operatorname{Mod}: M_{P}=0 \text { for all } P \in \Omega\right\} .
$$

In general we have $\mathcal{K}\left(\xi_{\Omega}\right)=\Omega$, and $\xi_{\mathcal{K}(\phi)}=\phi$ holds when $\mathcal{G}(\phi)$ has a basis of finitely generated ideals [5, Corollary 3.17].

Lemma 4.6. If $R$ is local with flat maximal ideal $M$, then $R$ is a valuation domain.

Proof. If $M$ is flat, $R / M$ is $\varphi$-torsionfree (Proposition 4.1) and hence $\tau$-torsionfree; this means that $\mathcal{K}(\tau)=\operatorname{Spec}(R)$ and hence $\tau=\xi_{\operatorname{Spec}(R)}=0$, which implies $\varphi=0$, and thus $R$ is a valuation domain by Proposition 4.1 and [13, Proposition 1.2].

Corollary 4.7. $\mathcal{K}(\varphi)=\mathcal{K}(\sigma)$.

Proof. Clearly $\mathcal{K}(\sigma) \subseteq \mathcal{K}(\varphi)$. For the converse inclusion note that if $P$ is a non-flat prime ideal and $P \hookrightarrow F$ is a flat envelope with $F \subseteq R$, then $P R_{P} \hookrightarrow F_{P}=R_{P}$ is 
a flat envelope over $R_{P}$, so that $P R_{P}$ is not $R_{P}$-flat. This, together with Lemma 4.6, shows that

$$
\mathcal{K}(\varphi)=\{P \in \operatorname{Spec}(R): P \text { is flat }\}=\left\{P \in \operatorname{Spec}(R): R_{P} \text { is a valuation ring }\right\}
$$

and thus $\mathcal{K}(\varphi)$ is generically closed. Now if $T$ is a $\varphi$-torsion module and $P$ is flat then $T_{P}$, as the cokernel of a flat envelope over $R_{P}$, is zero, and so $T$ is $\xi_{\mathcal{K}(\varphi)^{-}}$ torsion. As $\sigma$ is the smallest left exact radical bigger than $\varphi$, we get $\sigma \subseteq \xi_{\mathcal{K}(\varphi)}$ and therefore $\mathcal{K}(\varphi)=\mathcal{K}\left(\xi_{\mathcal{K}(\varphi)}\right) \subseteq \mathcal{K}(\sigma)$.

Theorem 4.8. Let $R$ be a commutative coherent ring with $\mathrm{w} \mathrm{D}(R) \leq 2$. Then $\varphi$ is a left exact radical.

Proof. By Proposition 4.2 we may assume that $R$ is local. As $\mathcal{Z}(\varphi)=\mathcal{Z}(\tau)$ and $\mathcal{K}(\varphi)=\mathcal{K}(\sigma)$, we just have to show that $\mathcal{Z}(\varphi) \cup \mathcal{K}(\varphi)=\operatorname{Spec}(R)$, for then $\mathcal{K}(\tau)=$ $\mathcal{K}(\sigma)$ and $\sigma \subseteq \xi_{\mathcal{K}(\sigma)}=\xi_{\mathcal{K}(\tau)}=\tau$, which implies $\tau=\varphi=\sigma$. So let $P$ be a nonflat prime ideal. We may find $a_{1}, \ldots, a_{n} \in P$ such that $d=\left[a_{1}, \ldots, a_{n}\right] \notin P$ by Proposition 4.4 (indeed we may take $n=2$ ), but then each $b_{i}=a_{i} / d$ belongs to $P$ and $\left[b_{1}, \ldots, b_{n}\right]=1$, so that $R b_{1}+\cdots+R b_{n}$ is a $\varphi$-dense ideal and $P \in \mathcal{Z}(\varphi)$.

As a consequence of Theorem 4.8 we give a new description of the monomorphic flat envelopes of $R$-modules. We say that a module $G$ is $\varphi$-injective if, for every exact sequence $0 \rightarrow L \rightarrow M \rightarrow N \rightarrow 0$ with $N \varphi$-torsion, each homomorphism $L \rightarrow G$ can be extended to $M$. And $G$ is $\varphi$-closed whenever it is $\varphi$-torsionfree and $\varphi$-injective; by Theorem 4.8, each flat module is $\varphi$-closed. Associated to each module $M$ we find a $\varphi$-closed module $M_{\varphi}$ (the module of quotients of $M$ ) and a map $\lambda: M \rightarrow M_{\varphi}$ such that each homomorphism from $M$ to a $\varphi$-closed module factors through $\lambda$ in a unique way (see [12, Chapter IX]).

Proposition 4.9. Let $M$ be a submodule of a flat $R$-module. Then the canonical map $\lambda: M \rightarrow M_{\varphi}$ is an essential flat envelope.

Proof. By hypothesis, $M$ has a monomorphic flat envelope $f: M \rightarrow F$ which extends to a homomorphism $f_{\varphi}: M_{\varphi} \rightarrow F$ such that $f_{\varphi} \circ \lambda=f$. As $M$ is $\varphi$ torsionfree, $\lambda$ is an essential monomorphism, and hence $f_{\varphi}$ is monic; this leads to a commutative diagram with exact rows:

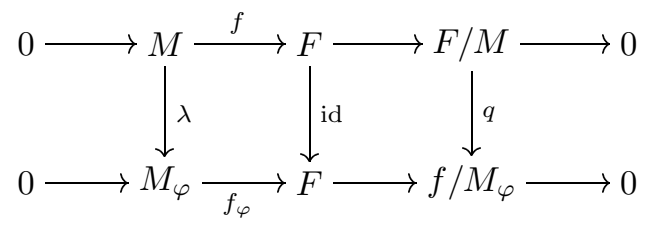

Since $F / M$ is $\varphi$-torsion and $q$ is epic, $F / M_{\varphi}$ is $\varphi$-torsion; but then the $\varphi$-injectivity of $M_{\varphi}$ forces $f_{\varphi}$ to be split and essential, so that it is an isomorphism.

Remark 4.1. We now have a way to construct the $\varphi$-torsion submodule of any module $M$ : Let $0 \rightarrow K \rightarrow F \rightarrow M \rightarrow 0$ be any exact sequence with $F$ flat, and let $K \rightarrow G$ be a flat envelope of $K$ with cokernel $T$; then we find monomorphisms $G \rightarrow F$ and $T \rightarrow M$ which give rise to the commutative diagram with exact rows

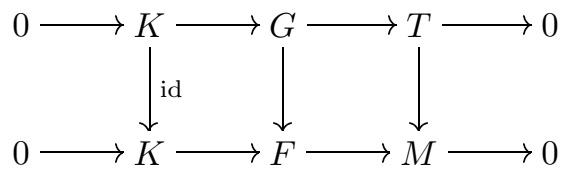


As $T \in \mathcal{T}(\varphi)$ and $M / T \cong F / G \in \mathcal{F}(\varphi), T$ must be the $\varphi$-torsion submodule of $M$.

We next investigate the structure of local coherent rings of weak global dimension 2. In [13], Vasconcelos showed that a commutative local ring of global dimension 2 (which is a coherent GCD-domain) must be a valuation domain, a regular local ring or, if neither of these, a so-called umbrella ring, which may be realized as the pull-back of two rings of the former types. For a local coherent ring $R$ with maximal ideal $M$ and weak global dimension 2 the situation is not so clear, although it is known that the dimension $k$ of the $(R / M)$-vector space $M / M^{2}$ can only take the values $k=0,1,2$, and that in case $k=2, M$ is generated by a regular sequence of length $2[13$, p. 54]. We present here a situation in which a sort of umbrella ring appears. Some of the ideas in the proof are taken from [13].

Theorem 4.10. Let $R$ be a coherent non-noetherian local ring of weak global dimension 2. If all non-flat prime ideals of $R$ are finitely generated, then there exists a prime ideal $P \neq 0$ such that $P=P R_{P}, R_{P}$ is a valuation domain and $R / P$ is a regular local ring of dimension 2.

Proof. As $\operatorname{wd}(R)=2, M$ cannot be flat by Lemma 4.6 and hence it is finitely generated but not principal, so that we are in the case $k=2$.

Now let $Q$ be a finitely generated prime ideal of $R$. We claim that each nonfinitely generated prime ideal is contained in $Q$. First note that $Q$ is either principal or non-flat, and in both cases all proper ideals strictly containing it are non-flat, so that $R / Q$ is a Noetherian ring by Cohen's theorem. Now let $P$ be a non-finitely generated prime; there exist $p_{1}, \ldots, p_{n} \in P$ such that $P+Q=R p_{1}+\cdots+R p_{n}+Q$ and, as $P$ is a directed union of principal ideals, we find $p \in P$ with $R p_{1}+\cdots+R p_{n} \subseteq$ $R p$ and thus $P+Q=R p+Q$. We shall show that $p \in Q$, and this will prove the claim. As $R p \neq P$, there exist $r \in M$ and $p^{\prime} \in P$ such that $p=r p^{\prime}$; on the other hand, $p^{\prime}$ must be of the form $p^{\prime}=t p+q$ for certain $t \in R$ and $q \in Q$, and therefore $p=(1-r t)^{-1} r q \in Q$.

Now pick an element $a \in M \backslash M^{2}$, which is prime since $R$ is a GCD-domain, and set $Q=R a$. Since $R_{Q}$ is a valuation domain by Corollary 4.7 and $Q$ contains all non-finitely generated primes, these are linearly ordered and its direct union $P \neq 0$ is still a flat prime ideal, so that $R_{P}$ is a valuation ring. It is also clear that $R / P$ is a noetherian ring of Krull dimension at least 2, as shown by the chain of primes $P \subset Q \subset M$, and the images in $R / P$ of a regular sequence of length 2 generating $M$ form a regular sequence generating $R / M$, which shows that $R / P$ is a regular local ring of dimension 2.

Remark 4.2. The prime spectrum of the ring $R$ of Theorem 4.10 looks like the prime spectrum of an umbrella ring, but note that if the global dimension of $R$ is $n>3$ (or if it is $n=3$ and the projective dimension of $R_{P} / P$ as an $R / P$-module is not 2), then $R_{P}$ is a valuation domain of global dimension $n$, and hence the non-finitely generated primes can be $\aleph_{n-2}$-generated by [10].

An instance of this case is given in [13, Example 4.12]: let $A$ be the localization of the polynomial ring $\mathbb{C}[X, Y]$ at the maximal ideal generated by the indeterminates, $Q$ the quotient field of $A$, and $R$ the subring of the power series ring $Q[[t]]$ consisting of those power series whose constant term lies in $A$. Then $R$ is as in Theorem 4.10, but it is not an umbrella ring since its global dimension is 3 . 


\section{REFERENCES}

1. J. Asensio Mayor and J. Martínez Hernández, Monomorphic flat envelopes in commutative rings, Arch. Math. 54 (1990) 430-435. MR 91k:16002

2. J. Asensio Mayor and J. Martínez Hernández, On flat and projective envelopes, J. Algebra 69 (1993) 434-440. MR 94k:16005

3. R. Belshoff, E.E. Enochs and J. Xu, The existence of flat covers, Proc. Amer. Math. Soc. 122 (1994) 985-991. MR 95b:16001

4. L. Bican, T. Kepka, and P. Němec, Rings, Modules and Preradicals, Lecture Notes in Pure and Applied Math. 75, Marcel Dekker Inc., New York, 1982. MR 83e:16026

5. J.L. Bueso, B. Torrecillas and A. Verschoren, Local Cohomology and Localization, Pitman Research Notes in Mathematical Series 226, Longman Scientific and Technical, Harlow, 1989. MR 93j: 13022

6. E.E. Enochs, Injective and flat covers, envelopes and resolvents, Israel J. Math. series 39 (1981) 189-209. MR 83a:16031

7. E.E. Enochs, Flat covers and flat cotorsion modules, Proc. Amer. Math. Soc. 92 (1984) 179184. MR 85j: 13016

8. J.L. Gómez Pardo and N. Rodríguez González, On some properties of IF rings, Quaestiones Math. series 5 (1983) 395-405. MR 85a:16015

9. J. Martínez Hernández, M. Saorín and A. del Valle, Noncommutative rings whose modules have essential flat envelopes, J. Algebra 177 (1995) 434-450. MR 96j:16003

10. B.L. Osofsky, Global dimension of valuation rings, Trans. Amer. Math.Soc. 127 (1967) 136149. MR 34:5899

11. J.D. Sally and W.V. Vasconcelos, Flat ideals I, Comm. Algebra 3 (1975) 531-543. MR 52:371

12. B. Stenström, An introduction to methods of ring theory, Grundlehren der mathematischen Wissenchaften, 217, Springer-Verlag, Berlin, 1975. MR 52:10782

13. W.V. Vasconcelos, The Rings of Dimension Two, Lecture Notes in Pure and Applied Math. 22, Marcel Dekker Inc., New York, 1976. MR 55:324

14. J. Xu, The existence of flat covers over Noetherian rings of finite Krull dimension, Proc. Amer. Math. Soc. 123 (1995) 27-32. MR 95c:16004

(Edgar E. Enochs) Department of Mathematics, University of Kentucky, Lexington, KENTUCKY 40506-0027

E-mail address: enochs@ms.uky.edu

(Juan Martínez Hernández and Alberto del Valle) Departamento de Matemáticas, UniverSidAd de Murcia, 30001 Murcia, Spain

E-mail address: juan@fcu.um.es

E-mail address: alberto@fcu.um.es 